\title{
Investigation of the Effects of the Incident Flow Angle on Vibration Behavior in Heat Exchanger Tube Bundle
}

\author{
Muhammad Usman ${ }^{1,2 *}$, Shahab Khushnood ${ }^{1}$, Luqman Ahmad Nizam", \\ Waqas Tanveer ${ }^{1}$, Ahmad Shafi ${ }^{2}$, Muhammad Ayub², \\ Hassan Farid Khan², Behzad Rustam² \\ 1 Department of Mechanical and Aeronautical Engineering, University of Engineering \& Technology Taxila, \\ 47050, Pakistan \\ 2 Mechanical Engineering Department, The University of Lahore, 1-KM Raiwind Road, 54000, Lahore, Pakistan \\ * Corresponding author's e-mails: muhammadusman3051@gmail.com
}

\begin{abstract}
An experimental study on the incident flow angle effects on the vibration behavior was carried out on aluminum tube in parallel triangular tube bundle with $\mathrm{P} / \mathrm{D}$ ratio of 1.375 . Fluid elastic instability is the most fatal mechanism from all the vibration mechanisms and therefore must be dealt with a lot of attention. Experiments were performed on low speed water tunnel with the velocity of water ranges from $0.3 \mathrm{~m} / \mathrm{s}$ to $0.7 \mathrm{~m} / \mathrm{s}$. The experiments were designed in a unique way to study the effects of incident flow angle on the vibration behavior. The monitored tube was mounted flexibly in an array of rigid tubes. The experiments were conducted on a flexible tube for different velocities ranging from $0.3 \mathrm{~m} / \mathrm{s}$ to $0.7 \mathrm{~m} / \mathrm{s}$ with different array rotated angles ( 0 to 90 degrees). It was observed that the vibration level was significantly high at 75 degree configuration as compared to other rotated angle configurations. It was also observed that the damping response is dispersed with all positive values, indicating that there is no instability in the tube.
\end{abstract}

Keywords: incident flow angle, fluid elastic instability, flow induced vibrations, parallel triangular array, crossflow velocity, flexible tube.

\section{INTRODUCTION}

For the last few decades, vibration in the steam generators and heat exchangers has been a major problem which affects the efficiency of the heat transfer process in utility industry. Shell side cross flow fluid forces act as an exciting mechanism which initiates tube vibration in the loosely supported tube bundle. Tube bundle vibration is an important concern for operators, designers, process engineer, as these vibrations result in mechanical damage such as tube fretting wear, tube collision damage, increase of baffle holes clearance, tube leakage or tube fatigue failure problems. Heat exchangers are equipped with baffles to increase thermal efficiency and support the tubes. In some processes, baffles are also used to direct the flow. Tubes are connected flexibly to a tube bundle with baffles. One fluid passes through the tubes and the other fluid passes through the tubes for heat transfer. The shell side fluid velocity also increases along with the heat transfer rate with the increased heat exchanger size. However, this also leads to vibrations. The amplitude of these vibrations depends greatly on the orientation of the tube relative to the flow, flow angle, pitch to diameter, damping conditions, mass ratio and tube material. If the amplitude of these vibrations becomes high, it will cause serious damage like fretting wear and necking failure of tubes. This leads the shutdown of heat exchanger and the whole process also demands expansive damage repair. Over the years, many researchers have studied the effects of these vibrations and developed the techniques to reduce their effect. Some of the techniques for dampening and reducing vibrations involve changing the $\mathrm{P} / \mathrm{D}$ ratio, changing the tube bundle orientation, changing the flow angle of approach to the bundle, 


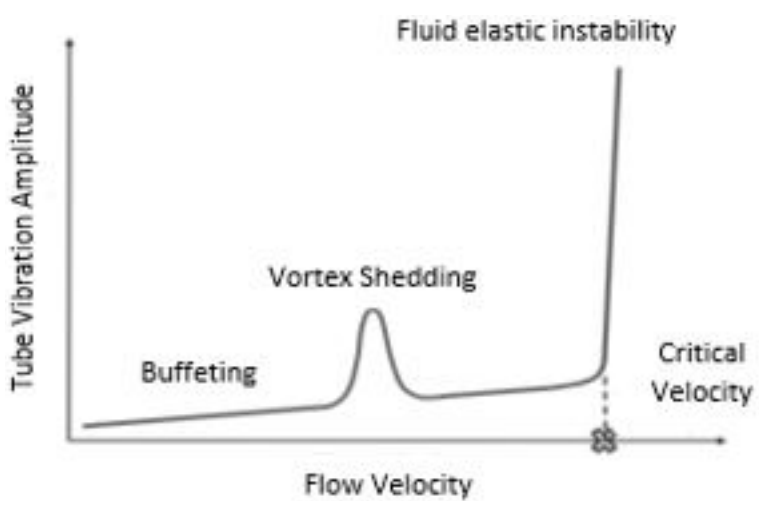

Fig. 1. Typical tube vibration response of a single tube in cross-flow [5]

changing the tube materials and changing the tube mass. Blevins [1] listed various non-dimensional parameters such as reduced velocity, Strouhal number, $\mathrm{P} / \mathrm{D}$ ratio, mass ratio, dimensionless amplitude, Reynolds number and damping, which were very critical in understanding the vibrations induced by the flow of tubes. For the hydraulic and thermal design of heat exchangers, Adelaja et al. [2] deliberately introduced a visual basic software design. They suggested that any small change in any design parameter such as tube length, baffle spacing ratio and tube bundle configuration could change the entire heat exchanger design.

Gawande et al. [3] found that the length of the tube has an enormous impact on the acoustic frequency of the shell side flow, the natural frequency of the tube, the bundle cross flow, the shedding ratio of the vortex and the critical velocity. In the literature, there are generally four different types of excitation mechanisms that result in damage to the tube bundle due to the vibrations induced by cross-flow [4]:

- Fluid-Elastic Instability.

- Vortex Induced Vibration.

- Turbulence Induced Excitation.

- Acoustic Resonance

Figure 1 presents the typical tube vibration response of a single tube subjected to cross-flow. The most important and destructive phenomenon that ultimately leads to a tube failure is fluidelastic instability. Instability is caused in a range of tubes by changing the fluid forces on the tube. Tanaka et al. stated that the tubes vibrate in oval orbits and the phenomena in heat exchangers are called fluid elastic instability [6]. Only one tube or one row of tubes was investigated in the early research on vibration of tube bundles. In this case, there is no FEI, only vibrations of the vortex shed-

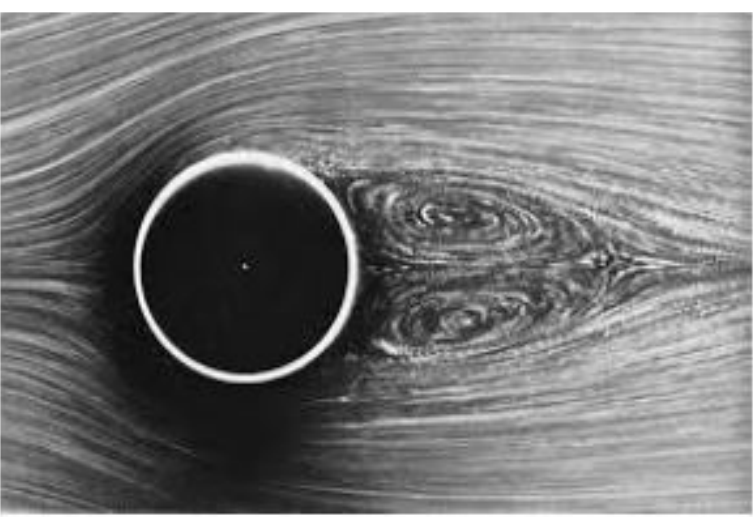

Fig. 2. Vortex shedding around a cylinder [8]

ding occurred (Figure 2). Roberts introduced the velocity mechanism, which states that instability is a time lag between the displacement of the tube and the imposition of the destabilizing fluid force. Roberts projected that this time lag is $(10 \mathrm{D} / \mathrm{U})$, $\mathrm{D}$ is tube diameter and $\mathrm{U}$ is the flow rate. Leaver et al. also concluded that $\mathrm{P}$ is pitch and $\mathrm{U}$ is the velocity between tubes $[1,7]$.

The displacement mechanism introduced by Connor states that instability is the result of the fluid forces generated by the interaction between adjacent tubes as they vibrate in synchronous oval orbits. Pettigrew et al. concluded that the eighth vibration mode in a multi-span heat exchanger tube bank before the fundamental tube mode became unstable in a particular case. This instability was the result of non-uniform flow distribution $[1,9]$. Weaver et al. concluded that instability depends on the geometry of the array and less on the spacing of the tube. Weaver et al. found that instability depends on the angle of the array geometry to a lesser extent when large amounts of data are assembled [10,11]. Andjelic et al. studied the effects of stability in the normal array of triangular tubes. The experimental results showed that there are two stability limits for a flexibly mounted cylinder in a stiff cylinder array. Minor variations in the array geometry were shown to affect its vibration mode [12]. Price et al. conducted experiments with multiple cylinders in cross flow. They concluded that instability is possible if three or more flexible cylinders are included in the first few rows of the array [13].

Khushnood et al. developed a review of vibrations in tube bundles induced by two-phase crossflow. They considered different combinations of fluids, flow regimes, dynamic parameters such as damping, added mass, different models of vacuum 


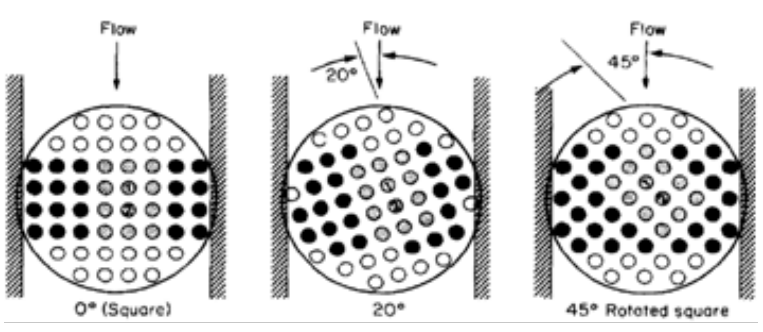

Fig. 3. Square bundle configuration at different angles [16]

fractions, measurement of vacuum fractions and their comparison, tube excitation mechanisms such as turbulence buffeting and elastic instability. They concluded that reliable design procedures can be developed by proper modeling and simulation of the vibrations induced by flow in the tube bundle [14]. Lin et al. carried out experiments in cylinder arrays on the cross-flow vibrations of a flexible cylinder. They concluded that the upstream cylinders have a significant impact on the flexible cylinder amplitude response. They increase the fluid elastic instability of a flexible cylinder and increase the cylinder vibration above the critical velocity, while the number of downstream cylinders had little effect on the vibration amplitude of the flexible cylinder. If the flexible cylinder has a different natural frequency than the surrounding cylinders, it has a minor effect on the critical velocity, but has a strong effect on the amplitude of the vibration above the critical velocity [15]. Khalifa et al. carried out experiments using a single flexible tube and in a tube array with a fully flexible tube. It was concluded that a single flexible tube in the third row of the rigid parallel triangular array of tubes becomes unstable at nearly the same velocity threshold as a fully flexible tube bundle. However, there is no instability in the single flexible tube in the first, second, fourth and fifth rows. It was also concluded that damping and stiffness of the tubes causes fluid-elastic instability [5].

Weaver et al. experimented with a square array of tubes. The array can be rotated to the flow at the transverse axis so that the changes in the approach flow angle and cross flow effects can be examined. Constant Strouhal number-based response to Vorticity was observed with some Strouhal number showing dependence on the angle of the incident. The angle of the incident flow has an effect on the elastic instability of the fluid [16].

Weaver et al. conducted experiments with water rigs on a normal triangular tube array. For ail orientations, a small response to vorticity amplitude was observed except for the parallel triangular array. Strouhal number of 0.57 was found based on pitch velocity and was found independent of the direction of the incident flow. In the normal triangular array, the fluid elastic threshold was found to be about twice that of the parallel triangular array [17]. Daniel et al. conducted an experimental study on triangular arrays of tubes with a ratio of 1.375 pitch to diameter. Rotated triangular arrays with $\mathrm{p} / \mathrm{d} 1.375$ are widely used in the industry, as it is a standard geometry and is used in CANDU power plants manufactured in Canada and used worldwide [18].

In this study, we conducted the experiments on parallel triangular tube array configuration while changing the applied flow angle to idealize the design requirements and optimize the permissible vibration limit to prevent fluid elastic instability. The experiments were conducted at different velocities at angles from 0 to 90 degrees.

\section{EXPERIMENTAL SETUP}

The vibrations of a flexible tube induced by cross-flow in a different range of rigid tubes were studied at different flow angles and at different velocities, keeping the pitch to diameter ratio constant at 1.375 . The tube consists of cylindrical tubes that form a parallel triangular configuration. The central tube remained flexible and had rigid tubes surrounding it. In terms of the fluid flow from the top section of the water tunnel, the bundle was transversely placed. Acrylic plates supported both ends of the tubes. The central tube was made flexible by using nylon strings to suspend it from both ends. Table 1 presents the tube bundle specifications.

Table 1. Specifications of Tube used in Array

\begin{tabular}{|l|c|}
\hline Material & Aluminum \\
Tube bundle configuration & Parallel triangular \\
Outer diameter & $12.7 \mathrm{~mm}$ \\
Inner diameter & $11.5 \mathrm{~mm}$ \\
Tube length & $180 \mathrm{~mm}$ \\
Number of tubes & 24 \\
P/D ratio & 1.375 \\
Modulus of elasticity & $69000 \mathrm{MPa}$ \\
Density of material & $2800 \mathrm{~kg} / \mathrm{m}^{3}$ \\
Mass of tube & $0.0115 \mathrm{~kg}$ \\
\hline
\end{tabular}



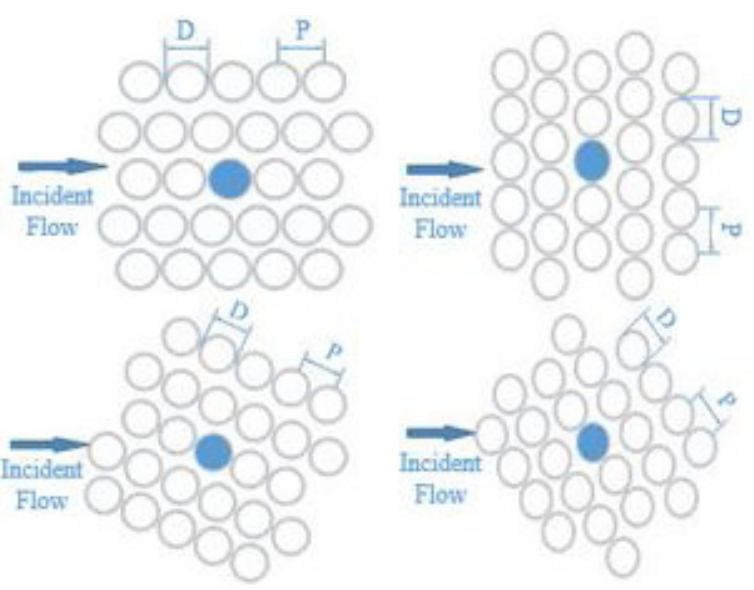

Fig. 4. Tube bundle configuration at different angles

Four types of tube bundle configuration are presented Figure 4, namely normal square, rotated square, normal triangular and rotated triangular (also known as parallel triangular).

The arrangement used to carry out the experiments involves the use of aluminum tubes and acrylic plates to make a parallel triangular tube bundle. In order to measure the effects of the fluid on it, a central tube was held flexible. Instability was observed by the accelerometer attached to the central tube in the central tube. A low-velocity water rig with a velocity of $0.3 \mathrm{~m} / \mathrm{s}$ to $0.7 \mathrm{~m} / \mathrm{s}$ was employed in the test section (Figure 5). The Doppler flow meter was used to measure the flow rate. The converging divergent part of the tunnel is metallic, while acrylic material is used for the test section. Water was pumped from a 200 gallon water tank by means of a $10 \mathrm{HP}$ centrifugal pump. The main valve (Valve 1) and the bypass

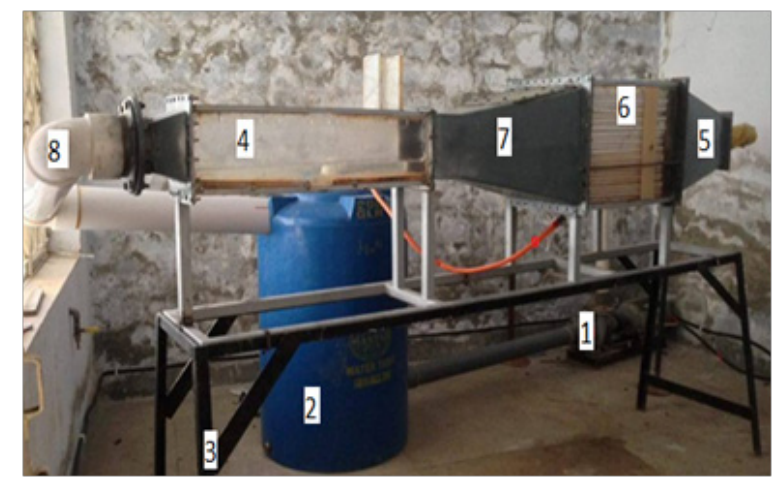

Fig. 5. Experimental setup (1 -10 HP motor with centrifugal pump; 2 - water tank; 3 - rig stand; 4 - test section; 5 - divergent section; 6 - flow straightener section; 7 - convergent section; 8 -discharge line)

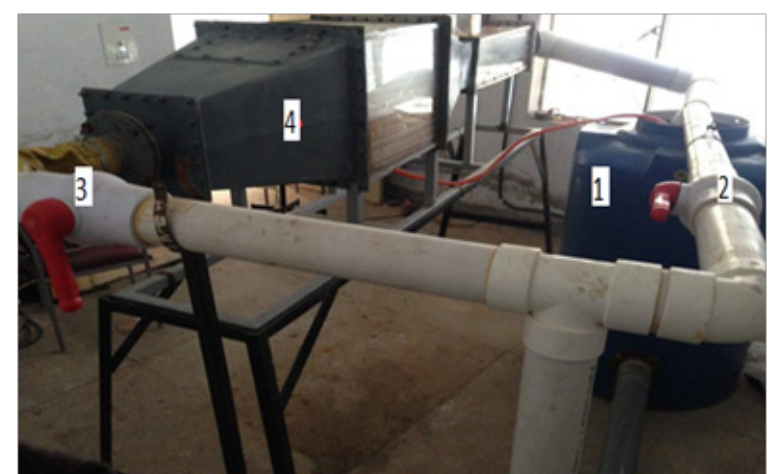

Fig. 6. Flow control mechanism (1 - water tank; 2 - valve $2 ; 3$ - valve $1 ; 4$-rig)

valve (Valve 2) are used (Figure 6) to control the water velocity in the test section. The flexible tube under consideration was mounted with a triaxial accelerometer to monitor the lateral (in the direction of flow) and transverse vibrations (perpendicular to flow). The natural frequency of the tube was tuned to $16-18 \mathrm{~Hz}$. The flexible tube was tightened to adjust the natural frequency of the flexible tube by means of a $0.5 \mathrm{~mm}$ thick piano wire and a tensioning mechanism.

The flexible tube was mounted in parallel triangular tube configurations, and the vibration response was observed at different velocities. The position of both valves (Valve 1 and Valve 2) was first adjusted for each measurement to achieve the desired velocity. The flow can then be stabilized at each velocity in the test section by giving 5 minutes and the data was then recorded. In order to avoid any errors, repeated measurements were carried out at the same velocity and different measurements were compared. For each velocity, the procedure was repeated until the maximum velocity was reached.

\section{Amplitude measurement}

The vibration amplitude was taken directly from the signal of the accelerometer. The response to RMS amplitude was taken by first taking the signal peak to peak value. The RMS amplitude $\left(\mathrm{A}_{\mathrm{rms}}\right)$ of the vibrating tube was calculated by using equation (1).

$$
A_{\text {rms }}=0.707 \frac{A_{p-p}}{2}
$$

Where, $A_{p-p}$ is the peak to peak amplitude, the resultant amplitude (A) is calculated by using relationship presented in equation (2). 


$$
A=\sqrt{A_{s w}{ }^{2}+A_{t r}^{2}}
$$

Where, $\mathrm{A}_{\mathrm{sw}}$ is Amplitude in stream-wise direction, $A_{t r}$ is Amplitude in transverse direction and $\mathrm{A}$ is Resultant Amplitude.

\section{Damping measurements}

Two methods were widely used for damping measurements, i.e. logarithmic decrease and bandwidth method. Both methods are effective for measuring damping. The logarithmic decrement method is shown in Figure 7 (a) and the bandwidth method is shown in Figure 7 (b). In the current analysis, both of these methods were used.

In order to calculate the damping factor $(\zeta)$ by using the logarithmic method, the equation (3) and equation (4) were used.

$$
\begin{gathered}
\delta=\frac{1}{n} \ln \left(\frac{x_{1}}{x_{n+1}}\right) \\
\zeta=\frac{\delta}{\sqrt{4 \pi^{2}+\delta^{2}}}
\end{gathered}
$$

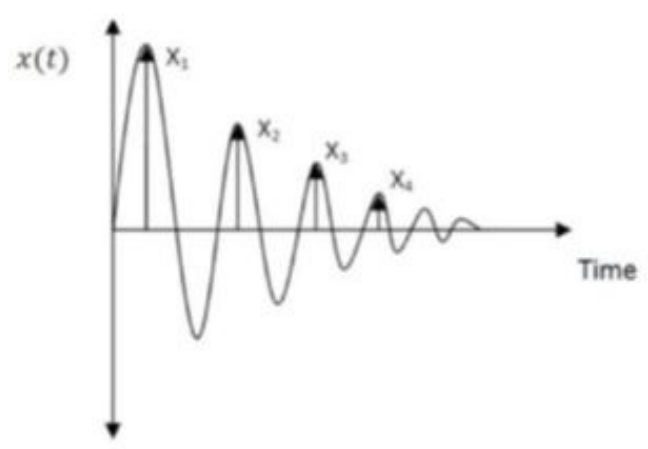

(a)

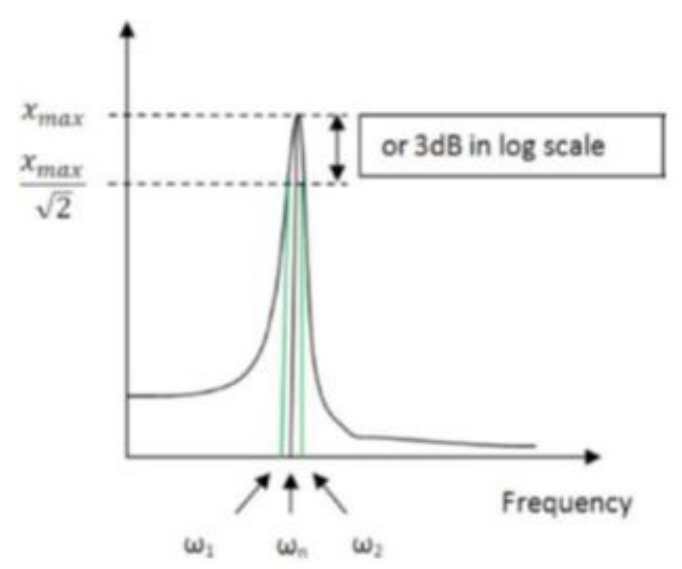

(b)

Fig. 7. (a) Logarithmic decrement method (b) Bandwidth method
Where, $x_{1}$ is Amplitude of first cycle and $x_{n+1}$ is Amplitude of the nth cycle.

Using the method of bandwidth, the equation (5) was used to calculate damping ratio in any medium in which the tube vibrates.

$$
\zeta=\frac{\omega_{2}-\omega_{1}}{2 \omega_{n}}
$$

Where, $\omega_{1}$ is the frequency of vibration $(\mathrm{rad} / \mathrm{s})$, $\omega_{2}$ is the frequency of vibration $(\mathrm{rad} / \mathrm{s})$ and $\omega_{\mathrm{n}}$ is the natural frequency of vibration $(\mathrm{rad} / \mathrm{s})$.

For slightly damped structures such as damping in tube bundle, equation (6) can be used to find the logarithmic decrement $(\delta$.

$$
\delta \cong 2 \pi \zeta
$$

The pitch velocity is required for data analysis, which is calculated using the equation (7) $[19,20]$.

$$
U_{p}=\frac{U P}{P-D}
$$

Where, $U_{p}$ is pitch or gap velocity $(\mathrm{m} / \mathrm{s}), U$ is free stream velocity $(\mathrm{m} / \mathrm{s})$, Pis center-to-center distance between two adjacent tubes and D is outer diameter of tube. The reduced velocity is calculated by using equation (8) [19].

$$
U_{r}=\frac{U_{P}}{f_{n} D}
$$

Where, $U_{r}$ is reduced velocity, $U_{p}$ is pitch or gap velocity, $f_{n}$ is natural frequency of tube, $D$ is outer diameter of tube.

In summary, the standard methods were adopted for different measurements. These measurements were then used for the data analysis. The complete analysis was discussed.

\section{RESULTS AND DISCUSSIONS}

\section{Incident flow angle Variations}

The Lift (y) and Drag (x) response for the instrumented tube is shown in Figure 8 and Figure 9 . The tube orientation at (0 degrees and 90 degrees) is in parallel triangular and normal triangular, respectively. Amplitude is seen to be approximately equal at zero degree angle for most velocities. However, as the angle increases, the amplitude differs from the same angle for different velocities. Drag shows more response to scatter than lift. This is because the drag response is more dominant at low fluid velocities than the pre-instability 


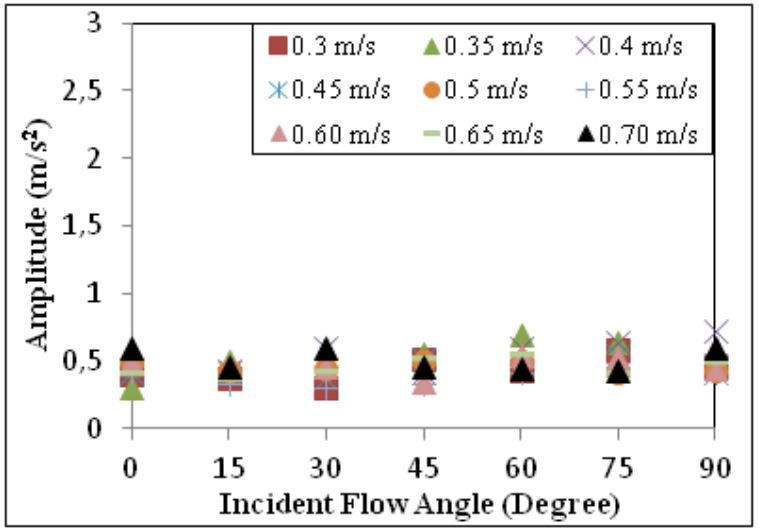

Fig. 8. Lift amplitude versus incident flow angle

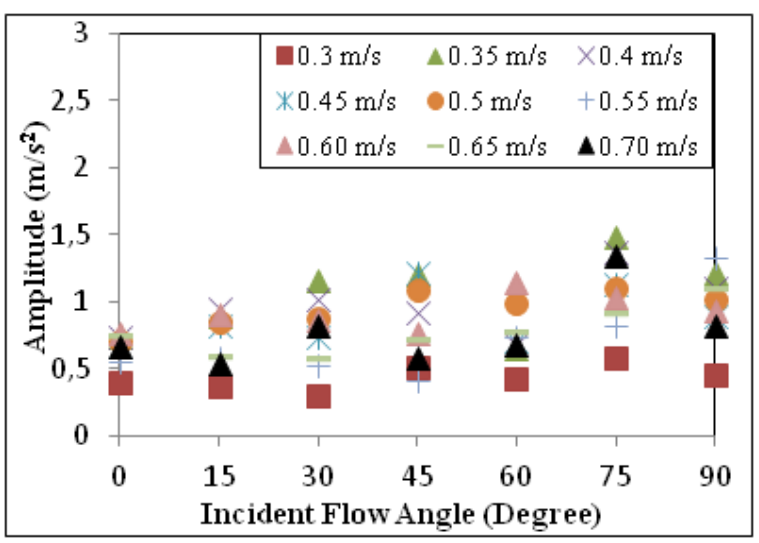

Fig. 9. Drag amplitude versus incident flow angle

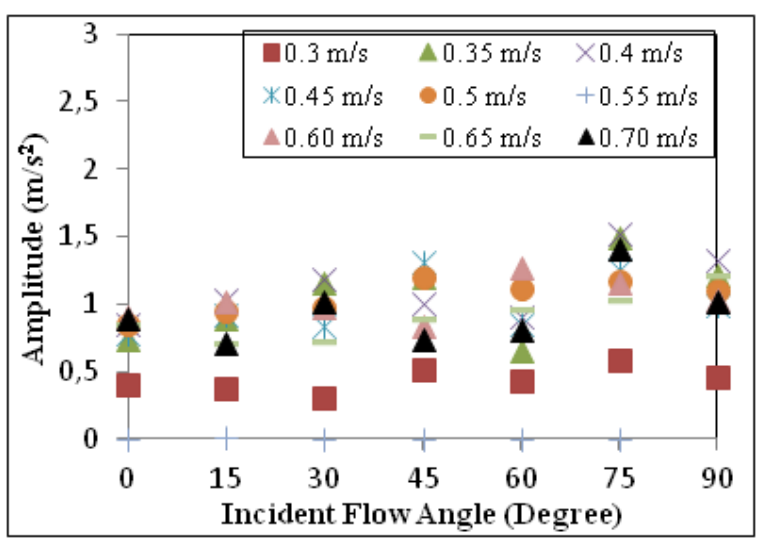

Fig. 10. RMS amplitude response versus Incident flow angle

lift response. The trend of amplitude response shows that fluid turbulence is the dominant source of excitement. Figure 10 shows the overall response of the RMS amplitude for the instrumented tube.

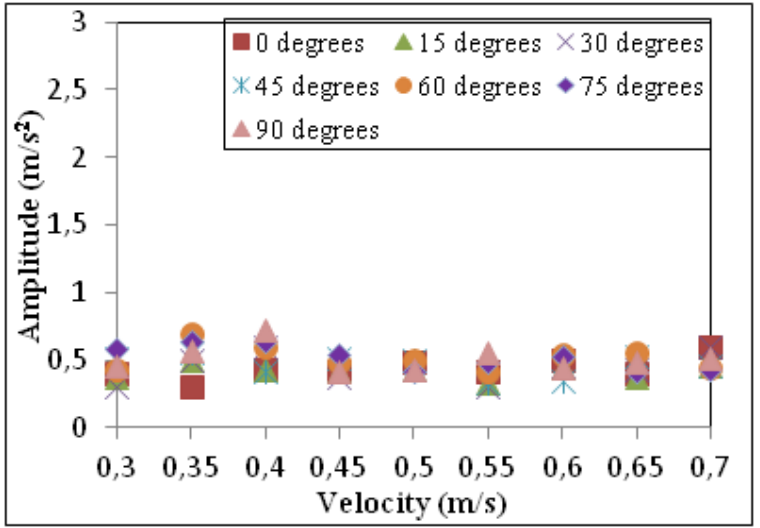

Fig. 11. Lift amplitude versus velocity

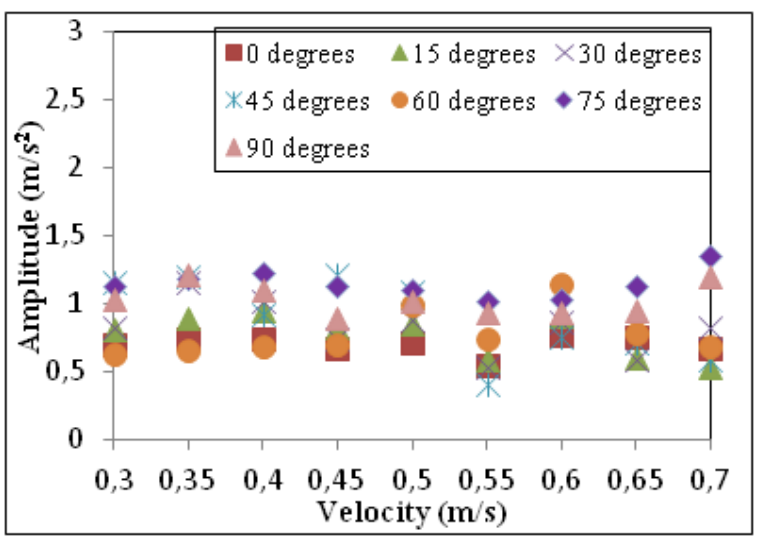

Fig. 12. Drag amplitude versus velocity

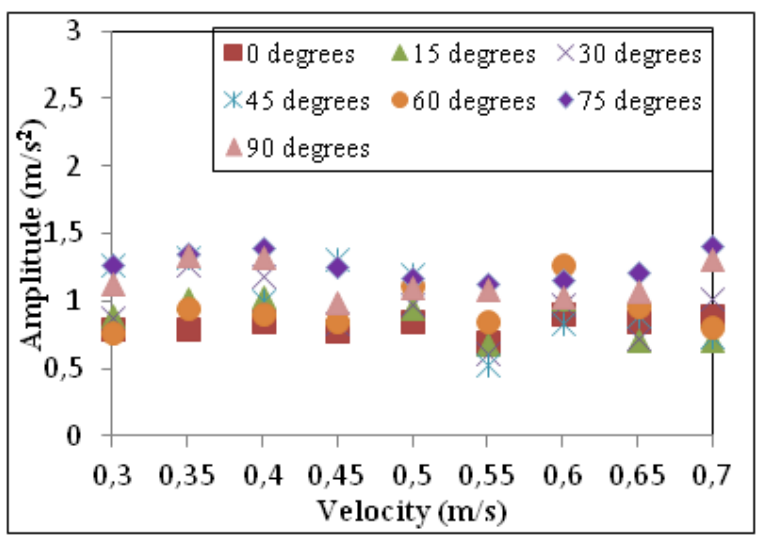

Fig. 13. RMS amplitude versus velocity

The overall lift and drag RMS amplitude response shows that the highest lift and drag amplitude occurs at 75 degrees. Since an overall linear amplitude trend is seen at an increasing angle, it is concluded that fluid turbulence is the dominant source of excitation. 


\section{Velocity variations}

The Lift and Drag response for the instrumented tube is shown in Figure 11 and Figure 12, while the fluid incident angle is constant and the velocity varies.

The Lift amplitude response of the instrumented tube in cross flow appears to be constant at a certain velocity for different angles, while the drag amplitude response seems to be dispersed at a specific velocity for different angles (Figure 11 and Figure 12). The higher amplitude level of the tube is also observed at an orientation of 75 degrees. This can be due to the tube orientation and the flow pattern in the bundle leading to high amplitudes (Figure 13). This response can also be seen in Figure 10, where the amplitude was highest at 75 degree orientation for different velocities.

\section{Damping}

In terms of damping, the energy dissipated as a structure vibrates. Damping is a very important phenomenon in the vibration analysis and vibration structure characterization. It depends heavily on the mechanical properties of the structure and the properties of the structure fluid. The damping response of the instrumented tube with variation in angle and velocity is shown in Figures 14 and 15.

There is a random damping response with an increase in the angle and velocity of the incident. The values show a small fluctuation from a constant trend. However, this random response appears to follow a linear trend that is the property of a turbulent excitement system. It is important to note that all damping ratio values are positive, indicating that there is no instability in the tube

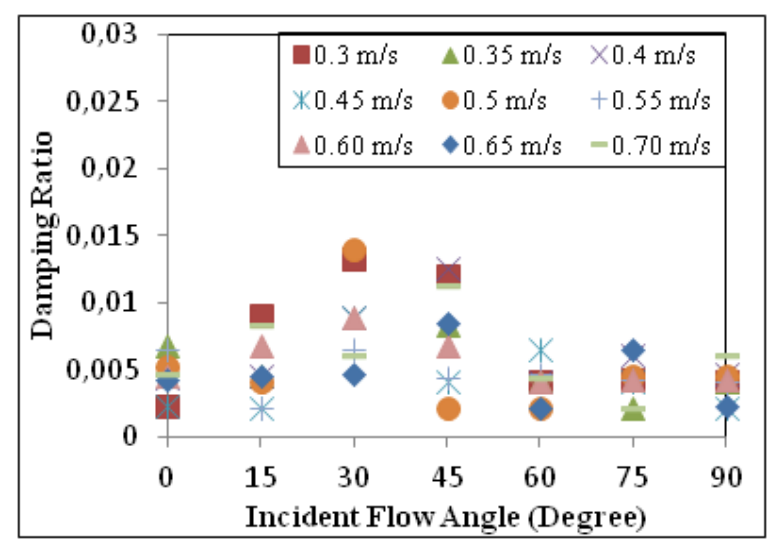

Fig. 14. Damping response of tube with variation in incident flow angle

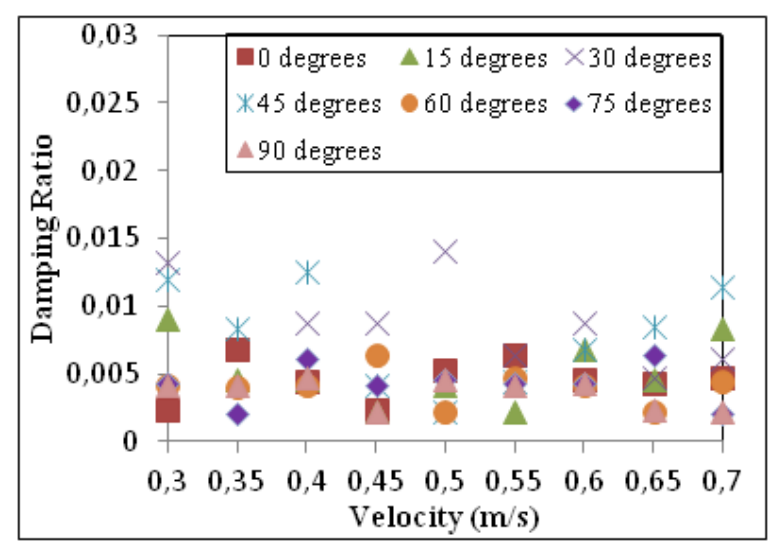

Fig. 15. Damping response of tube with variation in velocity

bundle for the current velocity range, since instability is characterized by a shift from positive to negative damping trends. Therefore, the turbulence excitement appears to be the dominant phenomena contributing to the tube vibration.

\section{Stability analysis}

The stability map is one of the most important criteria for predicting instability in a single cylinder and the fluid crossflow row of cylinder. Pettigrew and Taylor examined the fluid elastic instability for single-phase crossflow in detail. Fluid-elastic instability is mathematically formulated in terms of Mass-Damping Parameter (MDP) $\left(\mathrm{m} \delta\left(\rho \mathrm{D}^{2}\right)\right.$ and dimensionless or reduced velocity $\left(\mathrm{U}_{\mathrm{p}} /\left(\mathrm{f}_{\mathrm{n}} \mathrm{D}\right)\right.$ as presented in equation (9).

$$
\frac{U_{p c}}{f_{n} D}=K\left(\frac{m \delta}{\rho D^{2}}\right)^{a}
$$

Where $U_{p c}$ is the threshold velocity for fluid-elastic instability, $f_{n}$ is the natural frequency of the tube, $\mathrm{D}$ is the outer diameter of tube, $\mathrm{m}$ is the mass per unit length of the tube including the added mass, $\delta$ is the logarithmic decrement, $\rho$ is the density of the fluid, a is exponent to the mass-damping term and $\mathrm{K}$ is the Connor constant, which is a key parameter for predicting the stability boundary. There has been a lot of research to predict the value of $\mathrm{K}$ anda. It is generally agreed that the value of $\mathrm{K}$ is selected on the basis of the curve adjustment technique and that the value of a depends on the experimental conditions and generally amounts to 0.5 . The current experimental data on the stability map with the stability boundaries predicted by Price, Pettigrew and Taylor and Weaver and Fitzpatrick are presented in Figure 16. 


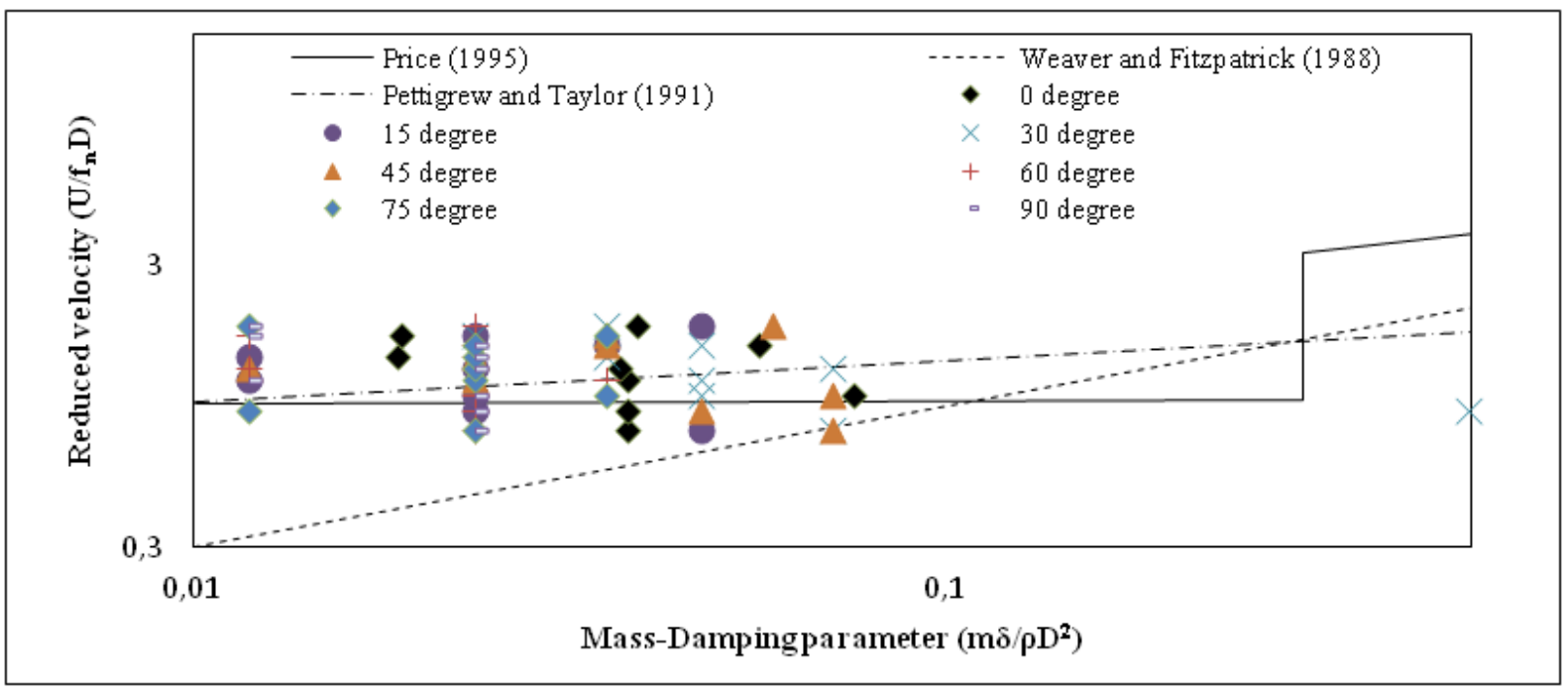

Fig. 16. Stability map of monitored tube for $P / D$ ratio of 1.375 with the stability boundaries predicted by Price, (Weaver and Fitzpatrick) and (Pettigrew and Taylor)

Figure 16 compares the current experimental data with the theoretical boundaries of stability predicted by Price [21], Weaver and Fitzpatrick [11] and Pettigrew and Taylor [22], which were found to be in good agreement with these theoretical boundaries of stability. Due to the low mass ratio, the narrow velocity range and the use of water as a working fluid, the most experimental data is concentrated on the left side of the map.

\section{CONCLUSIONS}

The following conclusions can be drawn from the current study:

1. Compared to other angle configurations, the 75 degree configuration offers a high level of vibration in cross flow, but is slightly less stable.

2. The vibration amplitude increases dramatically with the change in angle from (0 to 90 degrees), while the increase in amplitude with variation in velocity is stable.

3. The amplitude of the vibration increases along with velocity for different angle configurations, indicating that turbulence excitation is the dominant phenomenon contributing to the vibration generation.

4. The damping response shows a linear trend with all positive values that there is no instability in the tube.
5. The comparison of data with the theoretical stability boundaries (Figure 16) shows that the current experimental data are in good agreement.

\section{Acknowledgements}

The authors gratefully acknowledge the financial and technical support of the University of Engineering and Technology Taxila (UET, Taxila), University of Lahore (UOL) and Higher Education Commission (HEC) Pakistan.

\section{REFERENCES}

1. Blevins, R.D., Flow-induced vibration. 1990.

2. Adelaja, A., S. Ojolo, and G. Sobamowo, Computer Aided Analysis of Thermal and Mechanical Design of Shell and Tube Heat Exchangers. Advanced Materials Research, 2012, 367, 731-737.

3. Gawande, S.H., et al., Design Optimization of Shell and Tube Heat Exchanger by Vibration Analysis. Modern Mechanical Engineering, 2011, 1(01), 6-11.

4. Weaver, D., An introduction to flow induced vibrations "lecture notes. BHRA" The Fluid Engineering Center, Cranfield, Bedford England, 1993.

5. Khalifa, A., D. Weaver, and S. Ziada, A single flexible tube in a rigid array as a model for fluidelastic instability in tube bundles. Journal of Fluids and Structures, 2012, 34, 14-32.

6. Tanaka, H. and S. Takahara, Fluid elastic vibration of tube array in cross flow. Journal of sound and 
vibration, 1981. 77(1),19-37.

7. Lever, J. and D. Weaver, A theoretical model for fluid-elastic instability in heat exchanger tube bundles. Journal of Pressure Vessel Technology, 1982, 104(3),147-158.

8. Roberts, B.W., Low frequency, aeroelastic vibrations in a cascade of circular cylinders. Mechanical Science Monograph, 1966, (4).

9. Pettigrew, M., Y. Sylvestre, and A. Campagna, Vibration analysis of heat exchanger and steam generator designs. Nuclear Engineering and Design, 1978, 48(1), 97-115.

10. Weaver, D. and H. Yeung, The effect of tube mass on the flow induced response of various tube arrays in water. Journal of Sound and Vibration, 1984, 93(3), 409-425.

11. Weaver, D.T. and J. Fitzpatrick, A review of crossflow induced vibrations in heat exchanger tube arrays. Journal of Fluids and Structures, 1988, 2(1), 73-93.

12. Andjelic, M. and K. Popp, Stability effects in a normal triangular cylinder array. Journal of Fluids and Structures, 1989, 3(2), 165-185.

13. Price, S. and S. Kuran, Fluidelastic stability of a rotatedsquare array with multiple flexible cylinders, subject to cross-flow. Journal of fluids and structures, 1991, 5(5), 551-572.

14. Khushnood, S., et al., A review of heat exchanger tube bundle vibrations in two-phase cross-flow. Nuclear
Engineering and Design, 2004, 230(1), 233-251.

15. Lin, T.-K. and M.-H. Yu, An experimental study on the cross-flow vibration of a flexible cylinder in cylinder arrays. Experimental thermal and fluid science, 2005, 29(4), 523-536.

16. Weaver, D. and H. Yeung, Approach flow direction effects on the cross-flow induced vibrations of a square array of tubes. Journal of Sound and Vibration, 1983, 87(3), 469-482.

17. Yeung, H. and D. Weaver, The effect of approach flow direction on the flow-induced vibrations of a triangular tube array. Journal of Vibration and Acoustics, 1983, 105(1), 76-81.

18. Keogh, D.B. and C. Meskell, DRAFT: Bi-stable flow in parallel triangular tube arrays with a pitchto-diameter ratio of 1.375. Nuclear Engineering and Design, 2015, 285, 98-108.

19. Blevins, R.D., Flow-induced vibration. 1977: Van Nostrand Reinhold Company, New York.

20. Blevins, R.D., Flow-induced vibration. New York, Van Nostrand Reinhold Co., 1977, pp. 377.

21. Price, S., A review of theoretical models for fluidelastic instability of cylinder arrays in cross-flow. Journal of Fluids and Structures, 1995, 9(5), 463-518.

22. Pettigrew, M. and C. Taylor, Fluidelastic instability of heat exchanger tube bundles: Review and design recommendations. Journal of Pressure Vessel Technology, 1991, 113(2), 242-256. 